Article

\title{
Candidate Gene Selection for Cytoplasmic Male Sterility in Pepper (Capsicum annuum L.) through Whole Mitochondrial Genome Sequencing
}

\author{
Peng Wang ${ }^{1,2}$, Qiaohua $\mathrm{Lu}{ }^{1,2}$, Yixin $\mathrm{Ai}^{1,2}$, Yihao Wang ${ }^{1,2}$, Tiantian $\mathrm{Li}^{1,2}$, Lang $\mathrm{Wu}^{1,2}$, \\ Jinqiu Liu ${ }^{1,2}$, Qing Cheng ${ }^{1,2}$, Liang Sun ${ }^{1,2, *}$ and Huolin Shen ${ }^{1,2, *}$ \\ 1 Beijing Key Laboratory of Growth and Developmental Regulation for Protected Vegetable Crops, \\ China Agricultural University, Beijing 100193, China; wp737@outlook.com (P.W.); \\ lqh12261842@163.com (Q.L.); aiyixin0129@126.com (Y.A.); yhwang0906@126.com (Y.W.); \\ 1tt737737@163.com (T.L.); 15291832633@163.com (L.W.); 18811797502@163.com (J.L.); \\ chengqing2013@126.com (Q.C.) \\ 2 Department of Vegetable Science, College of Horticulture, China Agricultural University, \\ No. 2 Yuanmingyuan Xi Lu, Haidian District, Beijing 100193, China \\ * Correspondence: liang_sun@cau.edu.cn (L.S.); shl1606@cau.edu.cn (H.S.); \\ Tel.: +86-10-6273-1014 (L.S.); +86-10-6273-2831 (H.S.)
}

Received: 7 January 2019; Accepted: 22 January 2019; Published: 29 January 2019 updates

\begin{abstract}
Cytoplasmic male sterility (CMS), which is controlled by mitochondrial genes, is an important trait for commercial hybrid seed production. So far, genes controlling this trait are still not clear in pepper. In this study, complete mitochondrial genomes were sequenced and assembled for the CMS line 138A and its maintainer line 138B. The genome size of 138A is $504,210 \mathrm{bp}$, which is $8618 \mathrm{bp}$ shorter than that of 138B. Meanwhile, more than 214 and 215 open reading frames longer than 100 amino acids (aas) were identified in 138A and 138B, respectively. Mitochondrial genome structure of 138A was quite different from that of 138B, indicating the existence of recombination and rearrangement events. Based on the mitochondrial genome sequence and structure variations, mitochondrion of 138A and FS4401, a Korean origin CMS line, may have inherited from a common female ancestor, but their CMS traits did originate separately. Candidate gene selection was performed according to the published characteristics of the CMS genes, including the presence SNPs and InDels, located in unique regions, their chimeric structure, co-transcription, and transmembrane domain. A total of 35 ORFs were considered as potential candidate genes and 14 of these were selected, with orf300a and $0 r f 314 a$ as strong candidates. A new marker, orf300a, was developed which did co-segregate with the CMS trait.
\end{abstract}

Keywords: cytoplasmic male sterility (CMS); pepper (Capsicum annuum L.); mitochondria; CMS-associated gene

\section{Introduction}

Heterosis plays an important role in crop production. Hybrid crops produce $15-50 \%$ higher yields than the parent lines [1]. Therefore, heterosis has been widely used in the production of many cereal and horticultural crops, such as rice, maize, rapeseed, sorghum, sunflower, cucumber, watermelon, tomato as well as pepper [2]. However, one of the factors that limits the application of heterosis is emasculation, which is not only time-consuming and costly, but also unaffordable for many self-pollinating crops. Fortunately, the use of a male sterile line, which does not need emasculation during hybrid production, is a good solution for overcoming this problem. Since it 
was first successfully utilized in the production of hybrid corn (the maize CMS-T (Texas) system), the male-sterile-line-based hybrid technique has been applied in many other crops [3].

Cytoplasmic male sterility (CMS) is caused by sterility genes in the cytoplasm which lead to the abnormal stamen and incompatible pollen. Cytoplasmic male sterility can be restored by the restorer of fertility $(R f)$ gene in the nucleus. In contrast to genic male sterility (GMS), CMS is more widely used because the male-sterility trait can be maintained efficiently. Cytoplasmic male sterility is often found in higher plants and features maternal inheritance, pollen abortion, and normal pistil. It is often caused by the expression of a novel open reading frame (ORF) which is located on the mitochondrial genome [4,5]. Mitochondrial genomes of higher plants have many unique characteristics that are different from those of animals and fungi [6]. The most notable feature of the mitochondrial genome of higher plants is its genome size, which range from 200 to $2400 \mathrm{~kb}$ [7]. The mitochondrial genome of Brassica hirta is $208 \mathrm{~kb}$ [8], pepper $512 \mathrm{~kb}$ [9], and Silene 11.3 MB [10]. A large genome size of the plant mitochondrial genome often results in a large number of non-coding sequences, including gene spacing, repeat sequences, and introns. In addition, higher plant mitochondrial genomes usually show higher recombination and rearrangement rates, which could be attributed to the presence of repetitive sequences, which are considered to be one of the main drivers of the mitochondrial genome rearrangement [11-15]. Active recombination and rearrangement in plant mitochondrial DNA often lead to the generation of genes with altered ORFs, which are often manifested as aberrant chimeric structures or co-transcribed with genuine mitochondrial genes [5]. The expression of these aberrant chimeric genes at the RNA or protein level might affect the normal function of an inserted or co-transcribed gene and further affects the normal function of the mitochondria [11]. Those aberrant ORFs in higher plants are often considered as one of the main reasons for CMS.

Since the discovery of male sterility by the German botanist Joseph Gottlieb Kolreuter in 1763 [16], plants with male sterility have been identified in 43 families, 162 genera, and approximately 617 species [17]. More than 28 CMS-related genes have been identified in 13 crop species [3]. These genes often show chimeric structures with the mitochondrial genes present in the normal mitochondria, and encode proteins with transmembrane domains. For instance, the orf 79 and its variant orfH79 control the CMS-BT and CMS-HL in rice, respectively. Both of them encode proteins with chimeric structures, of which the $\mathrm{N}$ terminus sequences are similar to that of the $\operatorname{cox} 1$ gene and the remaining regions could not be aligned to any known proteins [18]. In radish, orf463 controls the CMS-DCGMS, of which the $5^{\prime}$ end 128 bp region can be aligned to cox 1 gene and the remaining region could not match any known genes [19]. Meanwhile, orf463 are predicted to contain 12 potential transmembrane domains. Although chimeric structure is one of the most notable features of the CMS genes, it is not an indispensable characteristic for the CMS gene. For example, in rice, orf352 has been confirmed to cause the CMS trait, which does not feature the chimeric structure, but co-transcribes with the ribosomal protein gene rpl5 [20].

The CMS sterility caused by novel mitochondrial ORFs can be restored by the $R f$ genes in the nucleus. Until now, more than ten $R f$ genes have been identified, such as $R f 2$ in maize [21,22], $R f 17$ in rice [23,24], Rf-PPR592 in Petunia [25], and PPR6 in pepper [26]. Most of the $R f$ genes are pentatricopeptide repeat (PPR) genes, which encode a group of RNA-binding proteins that often act in the organellar posttranscriptional mRNA processes, such as editing, splicing, cleavage, degradation, and translation $[27,28]$.

Pepper is an important vegetable crop and has been widely grown all over the world. Cytoplasmic male sterility has been widely used in hybrid pepper seed production. A number of studies have been conducted to identify candidate genes controlling CMS. So far, two candidate genes have been reported by a Korean group via restriction fragment length polymorphism (RFLP) analyses and RT-PCR [29-32]. One candidate, the atp 6 gene, encoding subunit of the mitochondrial ATP synthase complex, has been identified to be a gene duplication in the mitochondrial genome. The first copy of the atp6 gene, atp6-1, shared the same sequence in both of the male-sterile (Milyang-CMS) and maintainer lines (Milyang-N). However, in regard to the second copy of the atp 6 gene, atp6-2, there is a deletion at the $3^{\prime}$ end of 
the male-sterile allele ( $\psi$ atp6-2), which is considered to be the reason for the male-sterile trait $[29,31]$. Another candidate, identified in Milyang-CMS and 417/A, is the orf456/507 gene, which is called orf456 by Kim et al. [32], and orf507 by Gulyas [33]. The gene orf507 harbors a single nucleotide deletion at the +449 position which increases the ORF from $456 \mathrm{bp}$ to $507 \mathrm{bp}$ [32,33]. Recently, Jo et al. [9] published the complete mitochondrial genome sequence of the pepper CMS line FS4401 and the fertile line Jeju. orf507 and $\psi a t p 6-2$ were only identified in the mitochondrial genome of FS4401, further indicating they may be the candidates controlling CMS.

In this study, we sequenced and assembled the mitochondrial genomes of the CMS line 138A as well as its maintainer line 138B. A mitochondrial genome wide pairwise comparison was also conducted among the two Korean (FS4401 and Jeju) and two Chinese (138A and 138B) materials. Based on the genome sequence variation as well as the expression patterns, several candidate mitochondrial genes responsible for the male sterility in 138A were predicted.

\section{Results}

\subsection{Mitochondrial Genome Sequence of CMS Line 138 A and Its Maintainer Line $138 B$}

Mitochondrial genomes of CMS line 138A and its maintainer line 138B were sequenced via Illumina PE and PacBio techniques. For the Illumina PE sequencing, $5429 \mathrm{Mb}$ and $6837 \mathrm{Mb}$ clean data were obtained from line 138A and 138B, respectively. The Q30 values for 138A and 138B were $94.73 \%$ and $95.07 \%$, respectively. In regard to the PacBio sequencing, $98.36 \mathrm{Mb}$ filtered subreads with $\mathrm{N} 50=10,278 \mathrm{bp}$ and $101.83 \mathrm{Mb}$ filtered subreads with $\mathrm{N} 50=10,357 \mathrm{bp}$ were generated from line $138 \mathrm{~A}$ and $\mathrm{B}$, respectively. Through de novo assembling, mitochondrial genomes of 138A and 138B were obtained, of which the first one was $504,210 \mathrm{bp}$ and second one was $512,828 \mathrm{bp}$. The GC contents of the mitochondrial genomes of $138 \mathrm{~A}$ and $138 \mathrm{~B}$ were $44.55 \%$ and $44.51 \%$, respectively. The complete mitochondrial genome sequences of line 138A and 138B can be obtained in the GenBank nucleotide sequence database (https://www.ncbi.nlm.nih.gov/genbank/) under the accession numbers of MK225636 and MK225637, respectively.

\subsection{ORF Identification and Gene Annotation}

The ORF encoding proteins that were equal to or larger than 100 aas were identified from the assembled mitochondrial genomes. In 138A, 214 ORFs (including 33 known genes) and 34 non-coding RNAs (ncRNAs) were identified, and meanwhile 215 ORFs (including 33 known genes) and 31 ncRNAs were recognized in 138B (Supplementary Materials Table S1). Among those ORFs, 27 and 26 unique ORFs were identified in 138A and 138B, respectively (Supplementary Materials Tables S2 and S4). As to the known genes in 138A and 138B mitochondrial genomes, there was one-to-one correspondence with each other and encoded ribosomal protein large subunits RPL2, RPL5, RPL10, RPL16, RPS3, RPS4, RPS10, RPS12, RPS13, and RPS19; succinic acid dehydrogenase subunits SDH3 and SDH4; ATP synthase ATP1, ATP6, and ATP9; cytochrome oxidase subunits COX1, COX2, and COX3; cytochrome b COB; NADH dehydrogenase subunits NAD1, NAD2, NAD3, NAD4, NAD4L, NAD5, NAD6, NAD7, and NAD9; cytochrome $C$ synthesis related proteins CCMB, CCMC, CCMFC, and CCMFN; mature enzymes MATR. Finally, by combining with the mitochondrial sequences and ORFs identification results, mitochondrial genome maps of 138A and 138B were constructed (Figure 1). 

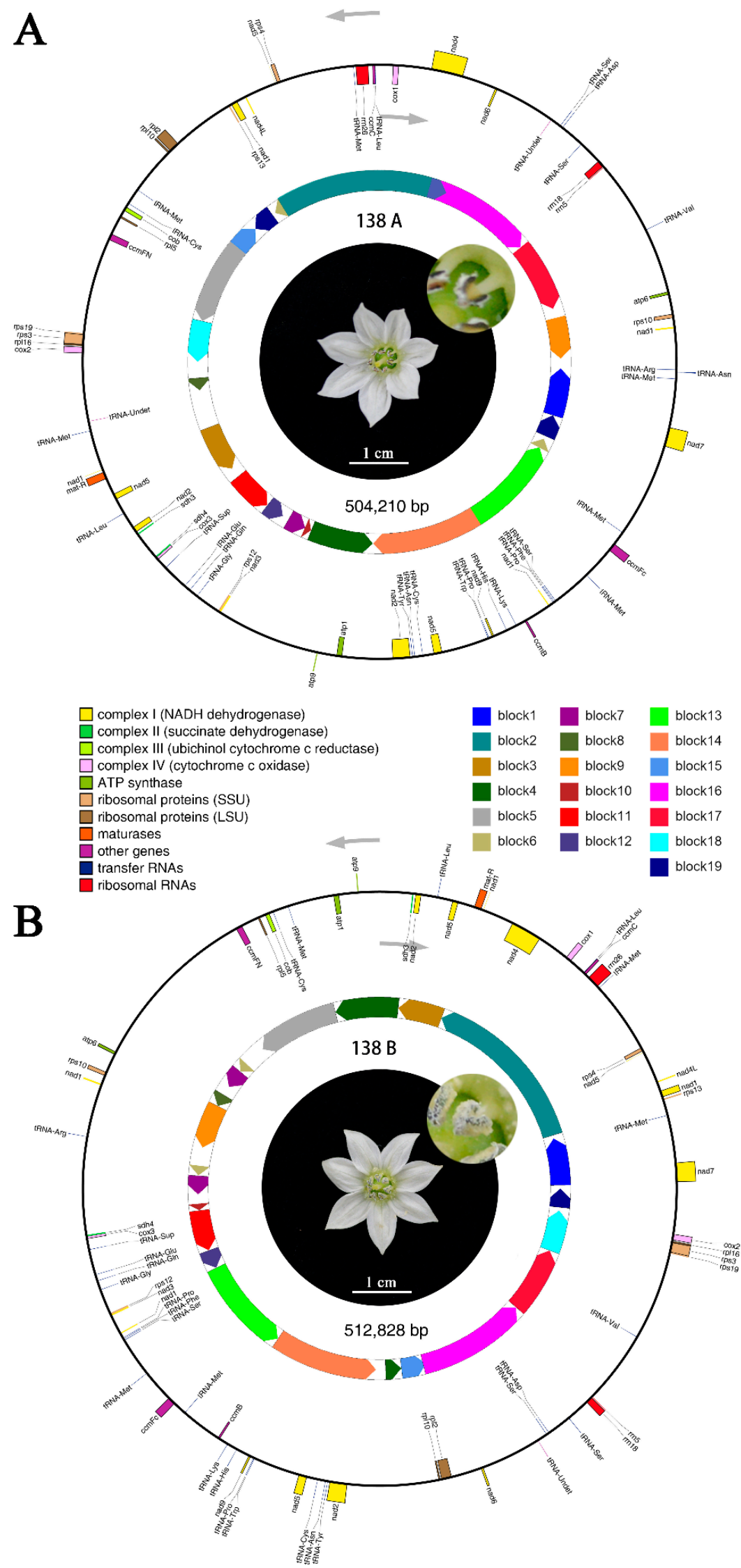

Figure 1. Mitochondrial genome maps of sterile line 138A and maintainer line 138B. (A) Mitochondrial genome map of 138A. (B) mitochondrial genome map of 138B. Genes with the names inside the circle 
are transcribed clockwise. Genes with names outside the circle are transcribed counterclockwise. The colors of the genes denote the functions of the gene products. Syntenic sequence blocks between genomes are depicted on the inner circles to separate blocks in different directions. In the center of the maps are the sterile flower of $138 \mathrm{~A}$ and the normal fertile flower of $138 \mathrm{~B}$, respectively.

\subsection{Comparative Analysis of the Mitochondrial Genomes}

\subsubsection{Syntenic Sequence Analysis of the Mitochondrial Genomes}

Comparative analysis was conducted between the of Korean CMS line FS4401 and its fertile line Jeju and CMS line 138A and maintainer line 138B. In terms of the genome size, 138A was $3242 \mathrm{bp}$ smaller than FS4401 (507,452 bp), while 138B was 1298 bp larger than Jeju (511,530 bp). In regard to the known functional gene annotation, compared with FS4401 and Jeju, both 138A and 138B lacked three genes, including atp 4 , atp 8 , and $m t t B$. A total of 19 syntenic sequence blocks were identified between 138A and 138B (Figure 1), which account for $91.68 \%$ and $90.24 \%$ of the mitochondrial genome sequences, respectively. A total of 23 unique regions ranging from $19 \mathrm{bp}$ to 10,469 bp were identified between 138A and 138B, of which 11 unique regions were found in 138A genome, and the other 12 unique regions were found in 138B genome (Supplementary Material Tables S3 and S5). In regard to the whole genome structure, there was an extensive recombination and rearrangement between the two mitochondrial genomes (Figure 2A). In contrast, a very high degree of collinearity, reaching more than $99 \%$, was observed between the CMS lines and fertility lines (Figure 2B,C).



Figure 2. Mitochondrial genome syntenic sequence analysis. (A) Syntenic sequence analysis of 138A and 138B; (B) syntenic sequence analysis of 138A and FS4401; (C) syntenic sequence analysis of 138B and Jeju. The upper and lower bars in each figure represented the mitochondrial genomes. Dark-orange and blue regions in each bar represented the forward and reverse direction of the aligned genome, respectively. White regions in each bar represent the sequences that could not be aligned to the other genome. Lines between the two bars indicated the syntenic types and locations: magenta, blue-green, dark yellow, and light-green represent for collinear, translocation, inversion, and tran + inver, respectively.

\subsubsection{Genome Structural Variation between 138A and 138B}

For further clarifying the difference between the two mitochondrial genomes, genome structural variations were investigated between $138 \mathrm{~A}$ and $138 \mathrm{~B}$ using $138 \mathrm{~B}$ as reference. As shown in Figure 3 , 13 ectopic, three inversion and eight ectopic + inversion regions were recognized between the two genomes (Figure 3). Meanwhile, two insertions and one deletion which were longer than $50 \mathrm{bp}$ were also identified in the syntenic regions. 


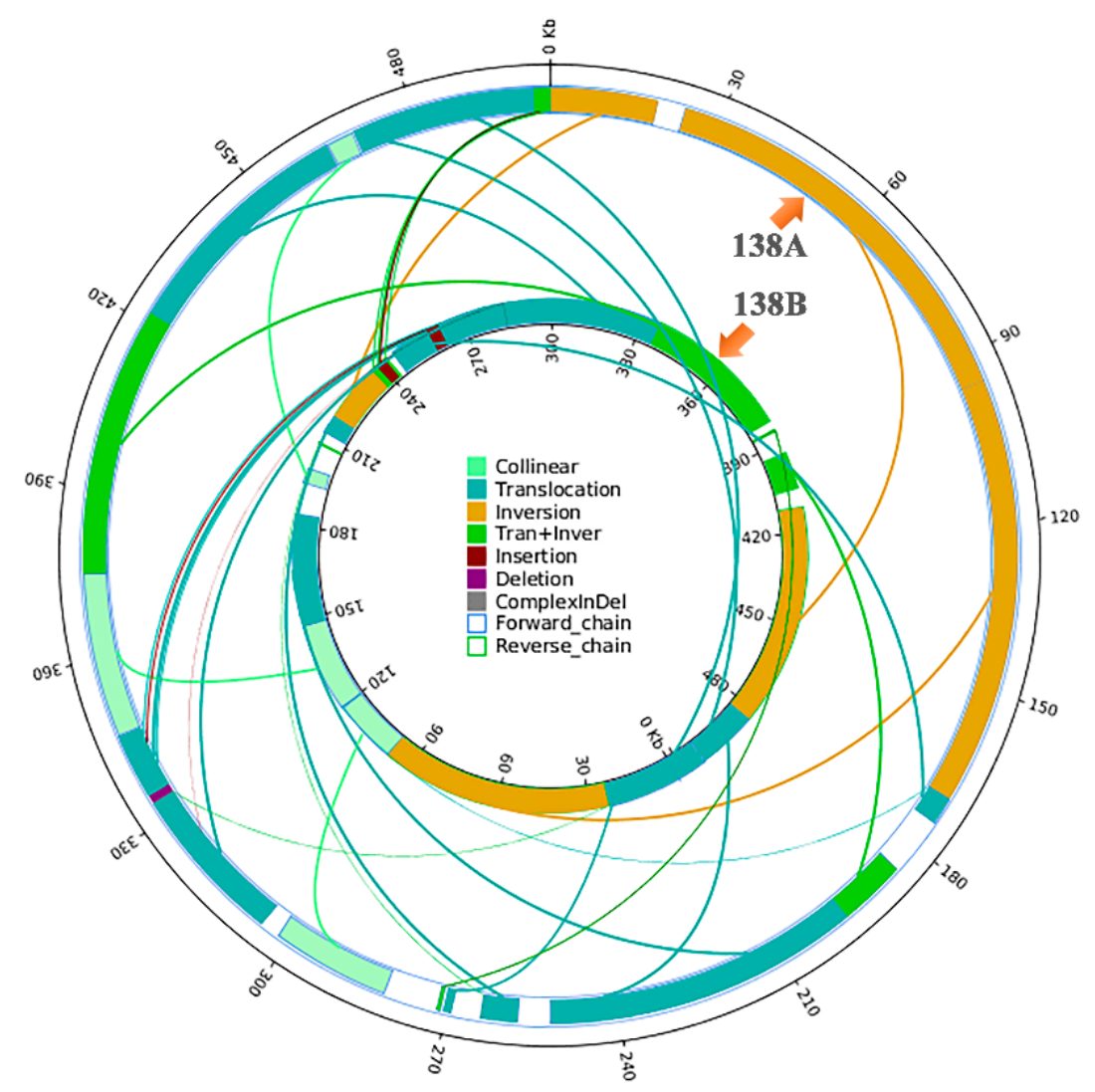

Figure 3. Structural variation map of mitochondrial genomes of 138A and 138B. The inner circle is the 138B genome, and the outer circle is the 138A genome. Collinear: the same linear region; Translocation: the area of translocation; Inversion: the area of inversion; Tran + Inver: the area of translocation and inversion; Insertion: the insertion region with a length greater than or equal to $50 \mathrm{bp}$; Deletion: the deletion region with length greater than or equal to $50 \mathrm{bp}$; Complex InDel: an area that cannot be compared, but corresponds to a position; Forward_chain: forward chain of the genome sequence, at which time the gene coordinates increase in a clockwise direction; Reverse_chain: the reverse chain of the genome sequence in which the genetic coordinates increase counterclockwise.

\subsubsection{SNP and InDel Detection}

In order to identify sequence variations in the known genes as well as the ORFs between 138A and 138B mitochondrial sequences, SNPs and InDels were detected between the two mitochondrial genomes. As shown in Tables S6-S8, a total of 112 SNPs and 14 InDels were identified between the two mitochondrial genomes. Among those mutations, only one SNP was identified in the known mitochondrial gene, mat-R; however, this SNP does not lead to non-synonymous mutations, suggesting it may not be the reason for the CMS. In another aspect, although seven SNPs which led to non-synonymous mutations were identified in six common ORFs shared by both 138A and 138B, no chimeric structure was identified in those ORFs (Supplementary Materials Table S11). In regard to the InDels, two were observed in two common genes shared by 138A and 138B; however, none of them resulted in the frame-shift mutation (Supplementary Materials Table S11).

\subsection{Selection of Candidate Genes Controlling the Cytoplasmic Male Sterility}

\subsubsection{Identification of Novel ORFs in the Unique Regions of 138A}

Based on previous studies, novel ORFs generated from the recombination and rearrangement of the mitochondrial genomes in the unique regions of CMS lines are often considered to be the genes controlling the CMS trait. In this study, twenty-seven 138A specific ORFs that encoded $\geq 100$ amino 
acids were identified (Supplementary Materials Table S2), of which 11 ORFs were located in the unique regions of 138A with full length or partial sequences (Supplementary Materials Table S3).

\subsubsection{Analyses of Chimeric Structures, Co-Transcript Event, and Transmembrane Domain}

It is known that most CMS and CMS candidate genes feature chimeric structures and/or co-transcribing with functional genes. Thus, chimeric structures and co-transcripts were analyzed with the 138A specific ORFs. To the chimeric structure, only one chimeric structure was predicted. This chimeric structure was found in orf292a, which contained whole $r p l 2$ exon 1 and a 7 bp sequence of unknown origin at the $3^{\prime}$ end (Figure 4G). Rpl2 encodes a ribosomal protein large subunit. The existence of the orf $292 a$ transcript was confirmed by RT-PCR and sequencing (Figure 5A). Another co-transcript event was only confirmed in orf300a and the nearby gene sdh3. This transcript consisted of the full length of orf300a and sdh3 as well as the $171 \mathrm{bp}$ intergenic sequence (Figure $4 \mathrm{~F}$ ). The co-transcript was also confirmed by RT-PCR (primers: orf300a_F/sdh3_R) and sequencing (Figure 5A). As transmembrane domain is another characteristic of the CMS genes, we performed structural analysis of 138A specific novel ORFs. The following five ORFs (orf157a, orf314a, orf262a, orf300a, and orf115b) were examined for the presence of transmembrane domains (Supplementary Materials Table S2, Figure 4A-E). Also, the expression patterns of four candidate genes, including orf157a, orf262a, orf300a, and orf $115 b$, were also analyzed in anthers of 138A and 138B using real-time PCR (Figure 6). Results showed that almost none of those four genes were expressed in 138B, suggesting those genes expressed at very low level in the maintainer line (Figure 6).
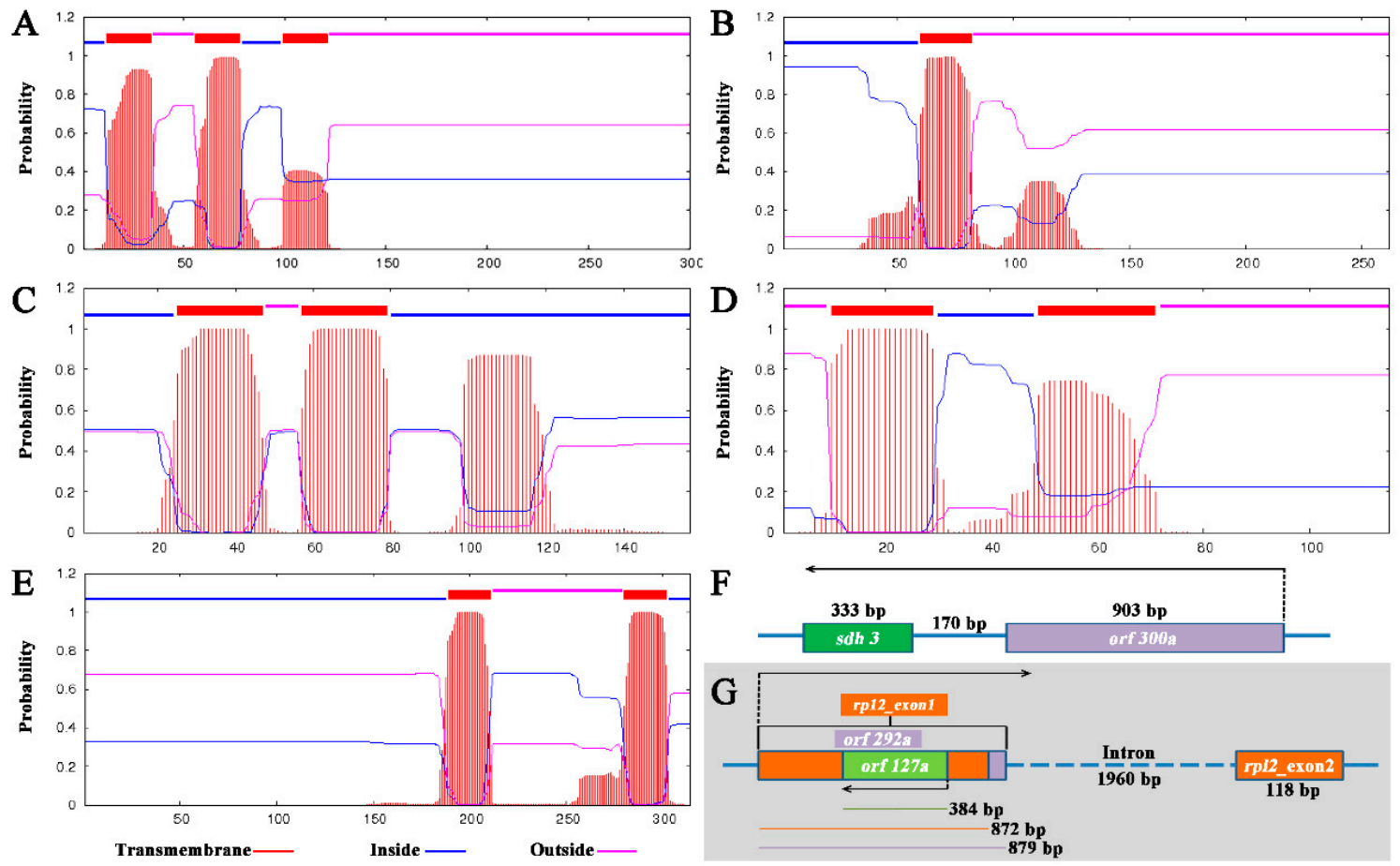

Figure 4. Chimeric structures, co-transcribe event, and transmembrane domain of the specific ORFs in 138A. (A-E): Locations and probabilities of the transmembrane domains of the gene products of orf300a (A); orf262a (B); orf157a (C); orf115b (D); and orf314a (E). (F) Co-transcribe structure of orf300a and $s d h 3 ;(\mathbf{G})$ chimeric structure of orf292a and rpl2_exon1, a long horizontal arrow shows orientation of transcription. 


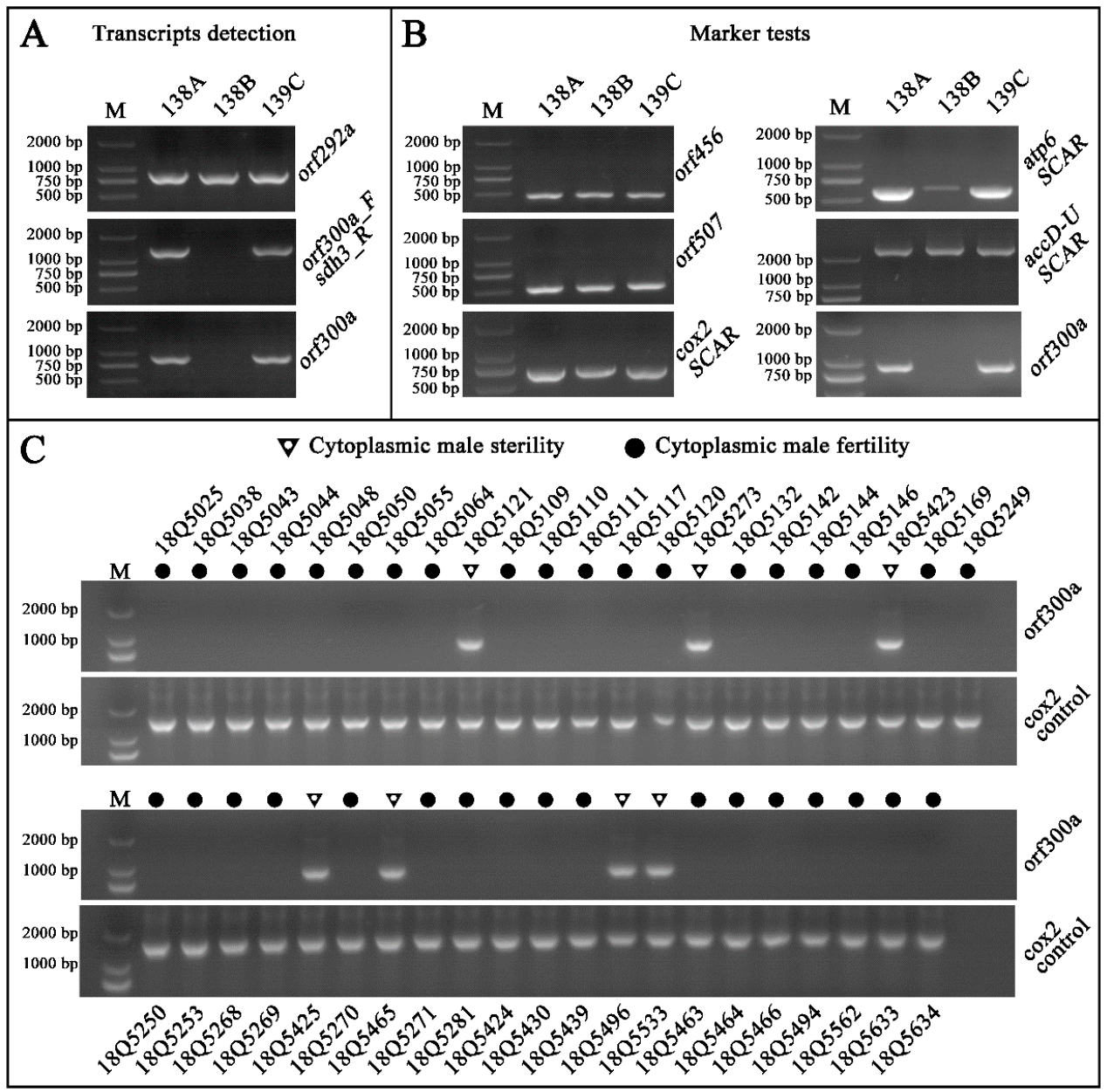

Figure 5. Results of PCR amplification. (A) Transcript detection of orf292a, co-transcript detection of orf300a-sdh3, transcript detection of orf300a; (B) CMS makers (orf456, orf507, cox2 SCAR, atp6 SCAR, accD-U SCAR, orf300a) test in 138A, 138B, 139C. (C) CMS maker orf300a tests in 43 pepper inbred lines, cox2 positive control.

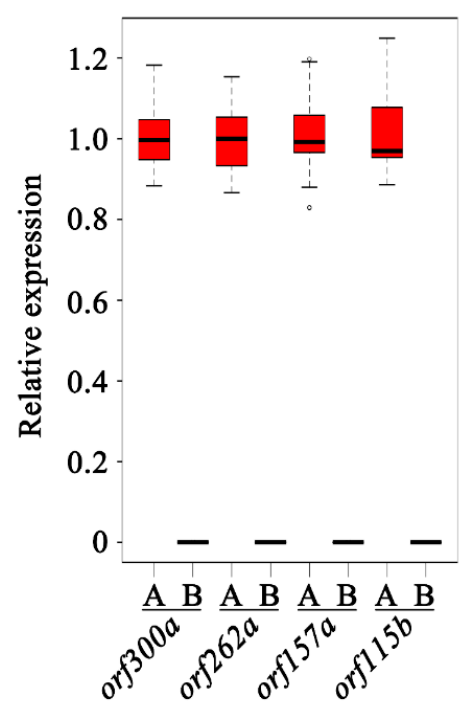

Figure 6. Transcriptional level of four 138A specific ORFs (orf262a, orf300a, orf157a, orf115b) in CMS line 138A and maintainer line 138B. Expression value the four ORFs in 138A was set as 1. UBI-3 was employed as the internal control. (A) and (B) represented lines 138A and 138B, respectively. 


\subsection{CMS Marker Development and Testing}

Identification of the CMS seedlings in the offspring is very important for the development of CMS lines. Molecular markers for the CMS trait are useful tools for accelerating the above process as well as the application of CMS in the $\mathrm{F}_{1}$ seed production. So far, at least five molecular markers have been developed for the identification of pepper CMS, including atp6 SCAR [30], cox2 SCAR [30], orf456 [32], orf507 [33], and acc D-U [34]. However, in our CMS lines and populations, they did not co-segregate with the CMS trait. Particularly, marker atp6 SCAR could not only be amplified in 138A, and 139C (contains sterile cytoplasm and the restorer of fertility $(R f)$ gene) lines, but also could be amplified in 138B (Figure 5B). Markers cox2 SCAR, orf456, orf507, and acc D-U did not show polymorphism among 138A, 138B, and 139C lines (Figure 5B). BLASTs result also supported the above observation that the primers sequences of marker cox 2 SCAR, orf 456 and orf507 could match the mitochondrial genomes of 138A and 138B very well. For marker acc D-U, primers did not align to genome sequences of 138A or 138B. Moreover, when those markers were used in the 43 pepper inbred lines which were developed in our lab from our CMS source (Supplementary Materials Table S9), no polymorphism was observed. Therefore, we tried to develop new markers for identifying the pepper CMS trait. Based on the unique ORFs in 138A, marker orf300a was developed. Further confirmation of this new marker was performed in the 43 pepper inbred lines which were developed in our lab and the male-sterile phenotypes of those lines were already known (Supplementary Materials Table S9). The results shown in Figure 5C indicated that the marker did completely co-segregate with the CMS trait, indicating this marker can be used to screen CMS plants which were originated from our CMS sources.

\section{Discussion}

\subsection{A and FS4401 May Originate from a Common Female Ancestor, but Their CMS May Originate Separately}

Through sequencing and de novo assembling, completed mitochondrial genomes of a pepper CMS line of Chinese origin (138A) and its maintainer line (138B) were constructed in this study. The mitochondrial genome sequence and structure of 138A was quite similar to that of FS4401, a previously reported Korean pepper CMS line, with the sequence variation rate and structure variation rate less than $2 \%$ and $1 \%$, respectively. Moreover, a similar phenomenon was also observed between the maintainer line 138B and a Korean local variety "Jeju", with the sequence variation rate and structure variation rate less than $2 \%$ and $1 \%$, respectively. For the CMS lines, although 138A was developed from a CMS plant which was discovered in the experimental field in China in 1986 [35] and the FS4401 was developed in Korea, their mitochondrial genome sequence and structure were highly similar to each other, suggesting they may originate from a common female ancestor. However, SNPs and InDels were identified between 138A and FS4401, and moreover, no difference was observed in orf507 between 138A and 138B, which is considered to be the gene controlling the CMS in FS4401, indicating that CMS of 138A was not transformed from FS4401 or its recent female parent, and vice versa. In regard to the maintainer line $138 \mathrm{~B}$ and the Korean local variety "Jeju", it is surprising that their mitochondrial genomes were also highly similar to each other. $138 \mathrm{~B}$ is an inbred line of C. annuum var. "Shanghaiyuan", which is an old local sweet pepper variety in the southeast of China. In regard to C. annuum var. "Jeju", according to the previous publications, it is a Korean local hot pepper variety [36]. Therefore, it is plausible that 138B and "Jeju" are two different varieties and it can be presumed that the variation of mitochondria genome in $C$. annuum or at least in some $C$. annuum varieties is small. A similar phenomenon was also reported in other Solanaceae species. In tomato the mitochondrial genome sequence of a wild tomato, Solanum pennellii, is $98 \%$ similar to that of the S. lycopersicum [37]. In conclusion, the mitochondrial genomes of 138A and FS4401 highly likely have been inherited from a common female ancestor but their CMS originated separately. 


\subsection{Candidate Gene Selection for the CMS Trait in $138 \mathrm{~A}$}

Cytoplasmic male sterility is an important trait for the hybrid seed production of both cereal and horticultural crops. So far, it has been confirmed that the CMS is not only caused by the SNPs and InDels, but also attributed to the rearrangement and recombination of the mitochondrial genome, which usually leads to the generation of new ORFs, chimeric structures, and co-transcribe events. Thus, for CMS candidate gene selection, the mitochondrial genome sequence variations as well as the mutations caused by the genome structural variations should all be considered. SNPs and indels leading to non-synonymous mutations and / or missing aas were detected in eight ORFs (Table 1), two of which were in the following known genes, orf 25 and orfB. Similarly, in tobacco a truncated orf 25 caused by an SNP was considered a strong candidate gene for CMS [38-40]. Thus, those eight genes with SNPs and InDels should be selected as potential candidate genes underlying the CMS. Also, new ORFs, chimeric structures, co-transcribe, and transmembrane domains are also important reasons for the CMS. For example, orf463, orf125, and orf138, which are the new ORFs generated by the rearrangement and recombination of the mitochondrial genome, are reasons for the CMS-DCGMS in radish, CMS-Kos in radish, and CMS-Ogu in Brassica, respectively [19,41,42]; in sorghum, orf107 encoding a chimeric protein with a segment of ATP9 at the $\mathrm{N}$ terminus is confirmed to cause CMS-A3 [43]; in sunflower, orf522 which is co-transcribed with the ATPA gene has been certified to induce the CMS [44]. In this work, 27 138A unique ORFs were identified which were all possible to cause the CMS and should be considered as the potential candidate genes. Furthermore, based on previous studies, most of the CMS genes have the chimeric structure and/or transmembrane domains, which lead to the disturbance of the normal function of mitochondria and finally cause the CMS. Meanwhile, those CMS genes are quite often found to be located on the unique regions of the CMS mitochondrial genomes. Therefore, those conditions were all considered in the further screen of the candidate genes in this study. Finally, 14 138A specific ORFs were found to have several or at least one of the following features: location on the unique region, chimeric structure, co-transcription, and transmembrane domain (Table 1). Among those 14 ORFs, orf292a was predicted to only have a chimeric structure; orf $115 b$ and orf $157 a$ were only considered to harbor transmembrane domains in their encoding products; orf $262 a$ and orf314a were predicted to not only have transmembrane domains, but also be located on the 138A unique regions; orf300a, which is located on the unique region of $138 \mathrm{~A}$, was predicted to not only have the transmembrane domains, but also co-transcribe with the sdh3 gene. Thus, all those 14 ORFs should be considered as the candidate genes for CMS in 138A. As orf300a met most of the conditions mentioned above, it was further selected as a strong candidate gene. Besides orf300a, orf314a was also considered to be a strong candidate for CMS gene. The sequence of orf $314 a$ in 138A was highly similar $(99 \%)$ to that of the atp6 in 138B; however, the length of orf314a (945 bp) was 351 bp shorter than that of the atp6. Meanwhile, atp6a in 138A was also highly similar (99\%) to that of atp6 in 138B, but the length of atp $6 a$ ( $855 \mathrm{bp}$ ) was $441 \mathrm{bp}$ shorter than of atp6 (Supplementary Materials Figure S1). atp6 encodes the ATP6 subunit of the mitochondrial ATP synthase and has been confirmed to be involved in the control of CMS. Therefore, it is high likely that orf314a and atp6a in 138A originated from the atp 6 gene. During the rearrangement and recombination of the mitochondrial genome, the original atp 6 in 138A did break into two pieces, and later, one piece evolved into apt6a and the other evolved into orf314a. This variation may lead to the disturbance of the normal function of atp6 and cause the CMS in 138A. Similar phenomenon was also reported by Kim et al. [31]. Finally, in this study, two ORFs, orf300a and orf314a, were selected as strong candidates for the CMS controlling gene in 138A. However, in the previous publication, orf507 was considered as a strong candidate gene for CMS in FS4401, because it was only found in the CMS line but not the MF line. Moreover, the short form of orf507, named orf 456 , has been confirmed to cause CMS via ectopic expression. In this work, orf507 was annotated in the mitochondrial genomes of 138A and 138B, and no sequence variation was found between the CMS and maintainer lines (Supplementary Materials Figure S2), suggesting orf507 should not be the candidate gene for CMS. 
Table 1. Features of the candidate genes controlling the CMS.

\begin{tabular}{cl}
\hline Features of the ORF & \multicolumn{1}{c}{ ORF ID } \\
\hline SNP/InDel & orf229a, orf138a, orf337a, orf675a, orf249a, orf104c, orfB, orf25 \\
On the unique region & orf108g, orf132a, orf314a, orf262a, orf165a, orf338a, orf244a, orf100b, orf300a, orf119a, orf100a \\
orf292a, orf300a \\
orf300a, orf314a, orf157a, orf115b, orf262a \\
Chimeric structure co-transcription \\
Transmembrane domain
\end{tabular}

\section{Materials and Methods}

\subsection{Plant Materials}

A pepper CMS line 138A and its maintainer line 138B were used in this study. 138A and 138B are in the sweet pepper "Shanghaiyuan" background. The CMS trait of 138A was transferred from an inbred line which originated from a CMS plant that was discovered in the production field of variety " $8633^{\prime \prime}$ in 1986. Approximately 1000 seeds were sown in a growth chamber with $28^{\circ} \mathrm{C}, 70 \%$ humidity, $16 \mathrm{~h} \mathrm{light} / 8$ darkness. Tender roots of the two lines were collected at the four-leaf stage, washed with tap water and frozen with liquid nitrogen, followed by storage at $-80^{\circ} \mathrm{C}$.

\subsection{Mitochondrial DNA Extraction}

Mitochondrial DNA was extracted using the GENMED large number of plant tissue mitochondrial DNA extraction kit (Genmed Scientifics Inc., Arlington, MA, USA). Pepper tender roots (60 g) were ground in a mortar under liquid nitrogen. The powdered tissues were then homogenized with $500 \mathrm{~mL}$ homogenization buffer (supply by the kit) in a glass homogenizer. The quality of DNA was assessed using NanoDrop2000, qubit3.0, and $0.8 \%$ agarose gel electrophoresis.

\subsection{Sequencing and Assembling of the Mitochondrial Genome}

One microgram of the purified DNA was fragmented to construct 430-bp short-insert libraries according to the manufacturer's instructions (Illumina, Hercules, CA, USA), and then sequenced on the Illumina Hiseq 4000 platform [45]. The high-molecular weight DNA was purified and used for PacBio library prep, BluePippin size selection, and then sequenced on the Sequel Sequencer.

Prior to assembly, the Illumina raw reads were filtered to remove the reads with adaptors, the reads showing a quality score below $20(Q<20)$, the reads containing a percentage of uncalled based (" $\mathrm{N}$ " characters) equal or greater than $10 \%$, and the duplicated sequences. The mitochondrial genome was reconstructed using a combination of the Pacbio Sequel data and the Illumina Hiseq data, and the following three steps were used to assemble the mitochondrial genomes. First, the genome framework was assembled by combing the Illumina and Pacbio data using SPAdes v3.10.1 [46]. Second, the assembly was verified and the circular or linear characteristics of the mitochondrial genome were completed, filling the gaps if any. Third, the clean reads were mapped to the assembled mitochondrial genome to correct the wrong bases and the presence of any insertion and deletion was judged. Finally, the complete mitochondrial genome sequences of 138A (CMS cytoplasm) and 138B (normal cytoplasm) were acquired and deposited in the GenBank nucleotide sequence database.

\subsection{Genome Component Analysis}

\subsubsection{Gene Annotation and Identification of ORFs}

The mitochondrial genes were annotated using homology alignments and de novo prediction. EVidenceModeler v1.1.1 was used to integrate the gene set [47]. The transfer RNA (tRNA) and ribosomal RNA (rRNA) genes were predicted by tRNAscan-SE [48] and rRNAmmer 1.2 [49], respectively. A whole mitochondria genome BLAST [50] (basic local alignment search tool) search (E-value $\leq 1 \times 10^{-5}$, minimal alignment length percentage $\geq 40 \%$ ) was performed against the following five databases: KEGG (Kyoto Encyclopedia of Genes and Genomes) [51-53], COG (Clusters 
of Orthologous Groups) [54,55], NR (Non-Redundant Protein Database databases), Swiss-Prot [56], and GO (Gene Ontology) [57]. The circular map of the mitochondrial genome was drawn using OrganellarGenomeDRAW v1.2 [58]. The prediction of the ORFs was undertaken using the software ORF Finder (http:/ / www.ncbi.nlm.nih.gov/gorf/gorf.html) if the hypothetical proteins encoded by the ORFs were longer than 100 amino acids. The names of the ORFs were given based on the number of amino acids that they encoded. Orf $x x+$ suffix $a / b / c: x x$ represents the length of the amino acid sequence of the orf, while the suffix $\mathrm{a} / \mathrm{b} / \mathrm{c}$ represents the same length, but different genes; orf $\mathrm{xx}+$ suffix $-1 /-2 /-3$ : $x x$ represents the length of the amino acid sequence of ORF, and the suffix $-1 /-2 /-3$ represents the duplicated genes of the same length.

The presence of a transmembrane domain in each hypothetical protein was predicted using TMHMM server v.2.0 (http:/ / www.cbs.dtu.dk/services/TMHMM/).

\subsubsection{Sequence Comparison between 138A and 138B}

In order to identify the syntenic sequence blocks and their translocation and inversion, a whole-genome synteny analysis was performed between 138A and 138B using the software programs MUMmer v3.23 and LASTZ v1.03.54. A single nucleotide polymorphism (SNP) was identified between $138 \mathrm{~A}$ and $138 \mathrm{~B}$ using the software programs MUMmer and BLAT v35. The size of the insertion-deletion (InDel) sequence ranging from 1 to $10 \mathrm{bp}$ was identified using the software programs LASTZ v1.03.54, BWA, and SAMtools. The structural variation (SV) of the two genomes was identified using the software programs MUMmer v3.23 and LASTZ v1.03.54.

\subsubsection{RT-PCR, Real-time PCR, and Sequencing of PCR Products}

Total RNA was isolated from different tissues and organs (root; stems; leaf; petals, anther, sepals and ovary at different times) of 138A, 138B, and 139C using an RNA extraction kit (SV total RNA isolation system, Promega), and the resulting RNA was reverse transcribed using the PrimeScript ${ }^{\mathrm{TM}}$ RT Reagent Kit (TaKaRa Bio Inc., Kusatsu, Shiga, Japan) to obtain $20 \mu \mathrm{L}$ of cDNA solution. The RT-PCR was performed in a 50- $\mu \mathrm{L}$ reaction volume containing $2 \mu \mathrm{L}$ template cDNA, $25 \mu \mathrm{L}$ high fidelity DNA polymerase (TaKaRa Bio Inc., Kusatsu, Shiga, Japan), $2 \mu \mathrm{L}$ forward primer $(10 \mu \mathrm{M}), 2 \mu \mathrm{L}$ reverse primer $(10 \mu \mathrm{M})$, and $19 \mu \mathrm{L}$ deionized water. The primers used in this study are listed in Supplementary Table S10. The RT-PCR was conducted using the following thermal cycles: initial denaturation at $94{ }^{\circ} \mathrm{C}$ for $5 \mathrm{~min}$; 28 cycles of $98^{\circ} \mathrm{C}$ for $10 \mathrm{~s}, 55^{\circ} \mathrm{C}$ for $15 \mathrm{~s}$, and $72{ }^{\circ} \mathrm{C}$ for $1 \mathrm{~min}$; then followed a final 5 min extension at $72{ }^{\circ} \mathrm{C}$. To add poly-A tails to PCR products, $10 \mu \mathrm{L}$ Taqmix was added to $50 \mu \mathrm{L}$ PCR product and the mixture was incubated at $72{ }^{\circ} \mathrm{C}$ for $30 \mathrm{~min}$. The amplification products were detected by electrophoresis on a 1.5\% agarose gel (containing gold view nucleic acid dye) and analyzed by UVI gel imaging system. The PCR products were purified using an Agarose Gel DNA Recovery Kit (GENERAY BIOTECH, Shanghai, China). The purified PCR product $(4 \mu \mathrm{L})$ was mixed with $1 \mu \mathrm{L}$ PMD19-T (TaKaRa Bio Inc., Kusatsu, Shiga, Japan) and $5 \mu \mathrm{L}$ ligase mix and incubated for $10 \mathrm{~h}$ at $16{ }^{\circ} \mathrm{C}$. Subsequently, the ligated product was sequenced by ABI PRISM 3730XL Analyzer (Applied Biosystems, Foster City, CA, USA).

The real-time PCR was performed using the TB Green ${ }^{\mathrm{TM}}$ Premix Ex Taq ${ }^{\mathrm{TM}}$ (TaKaRa Bio Inc., Kusatsu, Shiga, Japan), following manufacturer's instructions on an ABI 7500 real-time PCR system. The thermocycling conditions were set as follows: $95^{\circ} \mathrm{C}$ for $30 \mathrm{~s}, 40$ cycles of $95^{\circ} \mathrm{C}$ for $5 \mathrm{~s}$, and $60^{\circ} \mathrm{C}$ for $34 \mathrm{~s}$. Expression results were plotted based on $2^{-\Delta \Delta C t}$ method. Boxplot was generated via boxplot() in R.

\section{Conclusions}

Cytoplasmic male sterility is a very important phenotypic trait in pepper breeding. The present study reveals the complete genome sequences of the mitochondria in a pepper CMS line 138A cytoplasm and its maintainer line 138B normal cytoplasm. Those sequences can be found and downloaded in the GenBank nucleotide sequence database (https://www.ncbi.nlm.nih.gov/ 
genbank/) under the accession numbers of MK225636 and MK225637, respectively. By comparing the two mitochondrial genomes, several ORFs (orf300a, orf314a, orf262a, orf292a, orf157a, orf115b) that might be related to CMS, among them, orf300a and orf314a, were selected as strong candidates for the CMS controlling gene in 138A. In addition, orf300a has been successfully applied to CMS material screening as a molecular marker.

Supplementary Materials: Supplementary materials can be found at http:/ /www.mdpi.com/1422-0067/20/3/ $578 / \mathrm{s} 1$.

Author Contributions: P.W. performed most of the experiments, analyzed the data, and drafted the manuscript. L.S. made the figures and modified the manuscript. Q.L., Y.A., Y.W., and T.L. participated in mtDNA extraction. L.W., J.L., and Q.C. performed the RT-PCR and real-time PCR analysis. H.S. and L.S. designed and directed the entire study. All authors have read and approved the final manuscript.

Funding: This work is supported by The National Key Research and Development Program of China (2016YFD0101704) and Modern Agricultural Industry Technology System of Beijing Fruit Vegetables Innovation Team (BAIC01-2019).

Conflicts of Interest: The authors declare no conflict of interest.

\section{References}

1. Tester, M.; Langridge, P. Breeding technologies to increase crop production in a changing world. Science 2010, 327, 818-822. [CrossRef] [PubMed]

2. Li, S.; Yang, D.; Zhu, Y. Characterization and use of male sterility in hybrid rice breeding. J. Integr. Plant Biol. 2007, 49, 791-804. [CrossRef]

3. Chen, L.; Liu, Y.G. Male Sterility and Fertility Restoration in Crops. Annu. Rev. Plant Biol. 2014, 65, 579-606. [CrossRef] [PubMed]

4. Schnable, P.S.; Wise, R.P. The molecular basis of cytoplasmic male sterility and fertility restoration. Trends Plant Sci. 1998, 3, 175-180. [CrossRef]

5. Hanson, M.R.; Bentolila, S. Interactions of mitochondrial and nuclear genes that affect male gametophyte development. Plant Cell 2004, 16 (Suppl. 1), S154-S169. [CrossRef]

6. Knoop, V. The mitochondrial DNA of land plants: Peculiarities in phylogenetic perspective. Curr. Genet. 2004, 46, 123-139. [CrossRef] [PubMed]

7. Scheffler, I.E. Mitochondria; Wiley-Liss: New York, NY, USA, 1999.

8. Palmer, J.D.; Herbon, L.A. Unicircular structure of the Brassica hirta mitochondrial genome. Curr. Genet. 1987, 11, 565-570. [CrossRef] [PubMed]

9. Jo, Y.D.; Choi, Y.; Kim, D.H.; Kim, B.D.; Kang, B.C. Extensive structural variations between mitochondrial genomes of CMS and normal peppers (Capsicum annuum L.) revealed by complete nucleotide sequencing. BMC Genomics. 2014, 15, 561. [CrossRef]

10. Sloan, D.B.; Alverson, A.J.; Chuckalovcak, J.P.; Wu, M.; McCauley, D.E.; Palmer, J.D.; Taylor, D.R. Rapid evolution of enormous, multichromosomal genomes in flowering plant mitochondria with exceptionally high mutation rates. PLoS Biol. 2012, 10, e1001241. [CrossRef]

11. Budar, F.; Touzet, P.; De Paepe, R. The nucleo-mitochondrial conflict in cytoplasmic male sterilities revised. Genetica 2003, 117, 3-16. [CrossRef]

12. Kubo, T.; Newton, K.J. Angiosperm mitochondrial genomes and mutations. Mitochondrion 2008, 8, 5-14. [CrossRef] [PubMed]

13. Small, I.; Suffolk, R.; Leaver, C.J. Evolution of plant mitochondrial genomes via substoichiometric intermediates. Cell 1989, 58, 69-76. [CrossRef]

14. Albert, B.; Godelle, B.; Gouyon, P.H. Evolution of the plant mitochondrial genome: Dynamics of duplication and deletion of sequences. J. Mol. Evol. 1998, 46, 155-158. [CrossRef] [PubMed]

15. Woloszynska, M.; Trojanowski, D. Counting mtDNA molecules in Phaseolus vulgaaris: Sublimons are constantly produced by recombination via short repeats and undergo rigorous selection during substoichiometric shifting. Plant Mol. Biol. 2009, 70, 511-521. [CrossRef] [PubMed]

16. Mayr, E. Joseph Gottlieb Kolreuter's contributions to biology. Osiris 1986, 2, 76-135. [CrossRef]

17. Kaul, M.L. Male Sterility in Higher Plants; Springer: New York, NY, USA, 2012. 
18. Wang, Z.; Zou, Y.; Li, X.; Zhang, Q.; Chen, L.; Wu, H.; Su, D.; Chen, Y.L.; Guo, J.X.; Luo, D.; et al. Cytoplasmic male sterility of rice with boro II cytoplasm is caused by a cytotoxic peptide and is restored by two related PPR motif genes via distinct modes of mRNA silencing. Plant Cell 2006, 18, 676-687. [CrossRef]

19. Park, J.Y.; Lee, Y.P.; Lee, J.; Choi, B.S.; Kim, S.; Yang, T.J. Complete mitochondrial genome sequence and identification of a candidate gene responsible for cytoplasmic male sterility in radish (Raphanus sativus L.) containing DCGMS cytoplasm. Theor. Appl. Genet. 2013, 126, 1763-1774. [CrossRef]

20. Okazaki, M.; Kazama, T.; Murata, H.; Motomura, K.; Toriyama, K. Whole mitochondrial genome sequencing and transcriptional analysis to uncover an RT102-type cytoplasmic male sterility-associated candidate gene derived from Oryza rufipogon. Plant Cell Physiol. 2013, 54, 1560-1568. [CrossRef]

21. Cui, X.; Wise, R.P.; Schnable, P.S. The rf2 nuclear restorer gene of male-sterile T-cytoplasm maize. Science 1996, 272, 1334-1336. [CrossRef]

22. Liu, F.; Cui, X.; Horner, H.T.; Weiner, H.; Schnable, P.S. Mitochondrial aldehyde dehydrogenase activity is required for male fertility in maize. Plant Cell 2001, 13, 1063-1078. [CrossRef]

23. Fujii, S.; Komatsu, S.; Toriyama, K. Retrograde regulation of nuclear gene expression in CW-CMS of rice. Plant Mol. Biol. 2007, 63, 405-417. [CrossRef] [PubMed]

24. Fujii, S.; Toriyama, K. Suppressed expression of retrograde-regulated male sterility restores pollen fertility in cytoplasmic male sterile rice plants. Proc. Natl. Acad. Sci. USA 2009, 106, 9513-9518. [CrossRef] [PubMed]

25. Bentolila, S.; Alfonso, A.A.; Hanson, M.R. A pentatricopeptide repeat-containing gene restores fertility to cytoplasmic male-sterile plants. Proc. Natl. Acad. Sci. USA 2002, 99, 10887-10892. [CrossRef] [PubMed]

26. Jo, Y.D.; Ha, Y.; Lee, J.H.; Park, M.; Bergsma, A.C.; Choi, H.I.; Goritschnig, S.; Kloosterman, B.; van Dijk, P.J.; Choi, D.; et al. Fine mapping of restorer-of-fertility in pepper (Capsicum annuum L.) identified a candidate gene encoding a pentatricopeptide repeat (PPR)-containing protein. Theor. Appl. Genet. 2016, 129, 2003-2017. [CrossRef]

27. Delannoy, E.; Stanley, W.A.; Bond, C.S.; Small, I.D. Pentatricopeptide repeat (PPR) proteins as sequence-specificity factors in post-transcriptional processes in organelles. Biochem. Soc. Trans. 2007, 35, 1643-1647. [CrossRef] [PubMed]

28. Schmitz-Linneweber, C.; Small, I. Pentatricopeptide repeat proteins: A socket set for organelle gene expression. Trends Plant Sci. 2008, 13, 663-670. [CrossRef] [PubMed]

29. Kim, D.H.; Kang, J.G.; Kim, S.; Kim, B.D. Identification of cox II and atp6 region as associated to CMS in Capsicum annuum by using RFLP and long and accurate PCR. Hortic. Environ. Biotech. 2001, 42, 121-127.

30. Kim, D.H.; Kim, B.D. Development of SCAR markers for early identification of cytoplasmic male sterility genotype in chili pepper (Capsicum annuum L.). Mol. Cell 2005, 20, 416-422.

31. Kim, D.H.; Kim, B.D. The organization of mitochondrial atp6 gene region in male fertile and CMS lines of pepper (Capsicum annuum L.). Curr. Genet. 2006, 49, 59-67. [CrossRef]

32. Kim, D.H.; Kang, J.G.; Kim, B.D. Isolation and characterization of the cytoplasmic male sterility-associated orf456 gene of chili pepper (Capsicum annuum L.). Plant Mol. Biol. 2007, 63, 519-532. [CrossRef]

33. Gulyas, G.; Shin, Y.; Kim, H.; Lee, J.S.; Hirata, Y. Altered Transcript Reveals an Orf507 Sterility-Related Gene in Chili Pepper (Capsicum annuum L.). Plant Mol. Biol. Rep. 2010, 28, 605-612. [CrossRef]

34. Jo, Y.K.; Jeong, H.J.; Kang, B.C. Development of a CMS-specific marker based on chloroplast-derived mitochondrial sequence in pepper. Plant Biotechnol. Rep. 2009, 3, 309-315. [CrossRef]

35. Shen, H.L.; Jiang, J.Z.; Wang, Z.; Geng, S.S. Studies on the breeding and inheritance of male sterile lines of pepper (Capsicum annuum L.). J. Beijing Agric. Univ. 1994, 20, 25-30.

36. Lee, J.H.; An, J.T.; Han, K.; Choi, S.; Siddique, M.I.; Kang, B.C. Genetic mapping of resistance sources against ChiVMV (Chili veinal mottle virus) in hot pepper. Gene Genome New Technol. Plant Breed. 2016, 1, 220.

37. Wang, R.; Cai, X.H.; Fan, Y.J.; Hu, S.G.; Zhou, W. Advances in Solanaceae Mitochondrial Genomics. Genom. App. Biol. 2018, 1-10.

38. Heazlewood, J.L.; Whelan, J.; Millar, A.H. The products of the mitochondrial orf 25 and orfB genes are F0 components in the plant F1F0 ATP synthase. FEBS Lett. 2003, 540, 201-205. [CrossRef]

39. Liu, Q.; Zhu, T.; Zhao, T.; Huang, H. Aberrant mitochondrial gene orf25 may cause tobacco male sterility. Acta Agric. Univ. Jiangxiensi 2009, 31, 54-62.

40. Zhou, W.; Huang, H.; Zhou, B.N.; Liu, Q.Y. The bioinformatics analysis of orf25 gene related to tobacco cytoplasmic male sterility. Chin. Agric. Sci. Bull. 2011, 5, 312-316. 
41. Bonhomme, S.; Budar, F.; Lancelin, D.; Small, I.; Defrance, M.; Pelletier, G. Sequence and transcript analysis of the Nco2.5 Ogura-specific fragment correlated with cytoplasmic male sterility in Brassica cybrids. Mol. Gen. Genet. 1992, 235, 340-348. [CrossRef]

42. Tanaka, Y.; Tsuda, M.; Yasumoto, K.; Yamagishi, H.; Terachi, T. A complete mitochondrial genome sequence of Ogura-type male-sterile cytoplasm and its comparative analysis with that of normal cytoplasm in radish (Raphanus sativus L.). BMC Genom. 2012, 13, 352. [CrossRef]

43. Tang, H.V.; Pring, D.R.; Shaw, L.C.; Salazar, R.A.; Muza, F.R.; Yan, B.; Schertz, K.F. Transcript processing internal to a mitochondrial open reading frame is correlated with fertility restoration in male-sterile sorghum. Plant J. 1996, 10, 123-133. [CrossRef] [PubMed]

44. Köhler, R.H.; Horn, R.; Lössl, A.; Zetsche, K. Cytoplasmic male sterility in sunflower is correlated with the co-transcription of a new open reading frame with the atpA gene. Mol. Gen. Genet. 1991, 227, 369-376. [CrossRef] [PubMed]

45. Borgström, E.; Lundin, S.; Lundeberg, J. Large scale library generation for high throughput sequencing. PLoS One 2011, 6, e19119. [CrossRef]

46. Antipov, D.; Korobeynikov, A.; McLean, J.S.; Pevzner, P.A. HYBRIDSPADES: An algorithm for hybrid assembly of short and long reads. Bioinformatics 2016, 32, 1009-1015. [CrossRef] [PubMed]

47. Haas, B.J.; Salzberg, S.L.; Zhu, W.; Pertea, M.; Allen, J.E.; Orvis, J.; White, O.; Buell, C.R.; Wortman, J.R. Automated eukaryotic gene structure annotation using EVidenceModeler and the Program to Assemble Spliced Alignments. Genome. Biol. 2008, 9, R7. [CrossRef]

48. Lowe, T.M.; Eddy, S.R. tRNAscan-SE: A program for improved detection of transfer RNA genes in genomic sequence. Nucleic Acids Res. 1997, 25, 955-964. [CrossRef] [PubMed]

49. Lagesen, K.; Hallin, P.; Rødland, E.A.; Stærfeldt, H.H.; Rognes, T.; Ussery, D.W. RNAmmer: Consistent and rapid annotation of ribosomal RNA genes. Nucleic Acids Res. 2007, 35, 3100-3108. [CrossRef]

50. Altschul, S.F.; Gish, W.; Miller, W.; Myers, E.W.; Lipman, D.J. Basic local alignment search tool. J. Mol. Biol. 1990, 215, 403-410. [CrossRef]

51. Kanehisa, M.; Goto, S.; Kawashima, S.; Okuno, Y.; Hattori, M. The KEGG resource for deciphering the genome. Nucleic Acids Res. 2004, 32 (Suppl. 1), D277-D280. [CrossRef]

52. Kanehisa, M. A database for post-genome analysis. Trends Genet. 1997, 13, 375. [CrossRef]

53. Kanehisa, M.; Goto, S.; Hattori, M.; Aoki-Kinoshita, K.F.; Itoh, M.; Kawashima, S.; Katayama, T.; Araki, M.; Hirakawa, M. From genomics to chemical genomics: New developments in KEGG. Nucleic Acids Res. 2006, 34 (Suppl. 1), D354-D357. [CrossRef]

54. Tatusov, R.L.; Koonin, E.V.; Lipman, D.J. A genomic perspective on protein families. Science 1997, 278, 631-637. [CrossRef] [PubMed]

55. Tatusov, R.L.; Fedorova, N.D.; Jackson, J.D.; Jacobs, A.R.; Kiryutin, B.; Koonin, E.V.; Krylov, D.M.; Mazumder, R.; Mekhedov, S.L.; Nikolskaya, A.N.; et al. The COG database: An updated version includes eukaryotes. BMC Bioinform. 2003, 4, 41. [CrossRef] [PubMed]

56. Magrane, M. UniProt Knowledgebase: A hub of integrated protein data. Database 2011, 9. [CrossRef]

57. Ashburner, M.; Ball, C.A.; Blake, J.A.; Botstein, D.; Butler, H.; Cherry, J.M.; Davis, A.P.; Dolinski, K.; Dwight, S.S.; Eppig, J.T.; et al. Gene Ontology: Tool for the unification of biology. Nat. Genet. 2000, 25, $25-29$. [CrossRef]

58. Lohse, M.; Drechsel, O.; Bock, R. Organellar Genome DRAW (OGDRAW): A tool for the easy generation of high-quality custom graphical maps of plastid and mitochondrial genomes. Curr. Genet. 2007, 52, 267-274. [CrossRef] [PubMed]

(C) 2019 by the authors. Licensee MDPI, Basel, Switzerland. This article is an open access article distributed under the terms and conditions of the Creative Commons Attribution (CC BY) license (http:/ / creativecommons.org/licenses/by/4.0/). 